\title{
¿Es compatible el deporte en edad escolar con otros roles sociales? Un estudio a través de la Teoría de laAutodeterminación It is compatible the sport in scholar age with other social roles?
A study through Self-Determination Theory
}

\author{
Diana Amado Alonso, Francisco Miguel Leo Marcos, David Sánchez Oliva, \\ Inmaculada González Ponce, José María López Chamorro \\ Universidad de Extremadura
}

Resumen: El objetivo principal de este estudio fue examinar la relación entre la percepción de conflicto o utilidad del deporte respecto a otros papeles sociales y el nivel de autodeterminación en jóvenes deportistas, indagando sobre las diferencias que existen entre estas variables en función del género. Para ello, se utilizó una muestra de 1897 jugadores de diferentes disciplinas deportivas, 1378 de género masculino y 519 de género femenino. Los participantes rellenaron un cuestionario para medir el nivel de autodeterminación y otro para valorar el conflicto entre roles sociales. Los resultados mostraron que la utilidad del deporte se relacionaba positiva y significativamente con la motivación autoderminada mientras que el conflicto entre roles se relacionaba de esta misma forma con la desmotivación. Asimismo, los chicos mostraron puntuaciones elevadas en todos los tipos de motivación que abarca la teoría de la autodeterminación, siendo los que mayor incompatibilidad percibían entre el deporte y otros papeles sociales propios de su etapa de crecimiento. Como conclusión se destaca la necesidad de crear motivos de práctica intrínsecos en ambos géneros para favorecer el compromiso con la actividad deportiva y evitar el abandono que se puede producir por las exigencias de otros contextos que rodean al joven deportista. Palabra clave: conflicto entre roles, autodeterminación, práctica deportiva, edad escolar, género.

Abstract: TThe main aim of this study was to examine the relationship between perception of conflict or utility of the sport regarding other social roles and self-determination level in young athletes, focusing on differences among these variables with respect to sex. Hence, we used a sample size of 1897 players from different sport modalities, 1378 male and 519 female. Participants filled a questionnaire to assess self-determination level and other to measure conflict between social roles. Results showed that utility of sport was positive and significantly associated with self-determined motivation, whereas conflict between roles was related with amotivation. Moreover, male athletes showed higher scores in all types of motivation that refer SelfDetermination Theory, being the group that perceived the greater incompatibility between sport and other social roles according to their growth age. As a conclusion, we emphasize the necessity to promote intrinsic motives of practice in both sex to increase commitment with the sport activity, and avoid sport dropout that might be happened because of the demands of other contexts that surround young athletes.

Key words: Conflict between roles, self-determination, sport practice, scholar age, genre.

\section{Introducción}

Uno de los principales objetivos del deporte en edad escolar es crear hábitos de práctica deportiva para promover la formación integral del ser humano en sus dimensiones biológica, psicológica y social (Gutiérrez, 2004), adquiriendo una serie de conductas relacionadas con la salud que pueden mantenerse en la edad adulta (Castillo, 2000). Por ello, el estudio de las percepciones y comportamientos relacionados con la actividad física y el deporte en la población adolescente ha aumentado en los últimos años (Alvariñas, Fernández \& López, 2009; Amado, Leo, Sánchez-Miguel, Sánchez-Oliva \& García-Calvo, 2009).

En este sentido, resulta fundamental conocer la importancia que los jóvenes conceden a la práctica de actividad físico-deportiva dado que esto es lo que va a determinar el grado de interés y compromiso, evitando que se produzca un conflicto con otros roles sociales como el colegio, la familia, los amigos, etc., que puede conducir al abandono de dicha práctica (García-Calvo, Leo, Martín \& Sánchez, 2008; Guillet, Sarrazin, Carpenter, Trouilloud \& Cury, 2002). De hecho, las principales razones para no practicar deporte o para abandonar la práctica suelen ser la falta de tiempo, el cansancio después del trabajo o estudio, el querer probar otras tareas, la falta de interés (Rodríguez-Allen, 2000) e incluso en algunos trabajos se especifica claramente que el principal argumento es la falta de tiempo debido al nivel de exigencia académico (Edo, 2001; Rodríguez-Allen, 2000; Pavón, 2004).

Al respecto, tras analizar la literatura existente sobre esta problemática, algunos autores han demostrado que el hecho de poseer una motivación más autodeterminada hacia una actividad,es decir, realizar una actividad por voluntad propia y por el mero placer que reporta, hace que las personas sean menos susceptibles de percibir conflicto

Fecha recepción: 23-10-11 - Fecha envío revisores: 23-10-11 - Fecha de aceptación: 07-11-11 Correspondencia: Diana Amado Alonso

Avenida de la Universidad $\mathrm{s} / \mathrm{n}$

Avenida déceres

E-mail: diamal@unex.es entre dicha actividad y otros roles sociales. En cambio, cuando la motivación es menos autodeterminada, el sujeto es más susceptible de experimentar este conflicto (Boiché \& Sarrazin, 2007). Por este motivo, se ha optado por realizar esta investigación bajo el enfoque de la Teoría de la Autodeterminación (Deci \& Ryan, 2000; Ryan \& Deci, 2000), que es una de las teorías motivacionales más actuales. Dicha teoría, establece que la motivación es un continuo con diferentes niveles de autodeterminación que abarcan desde la conducta más autodeterminada hasta la menos autodeterminada, de modo que el recorrido de una a otra comprende tres tipos diferentes de motivación: la motivación intrínseca, que se asocia a mayores niveles de autodeterminación e implica el compromiso en una actividad por el placer que se obtiene al realizarla; la motivación extrínseca, que incluye cuatro tipos de regulación (integrada, identificada, introyectada y externa, pero dado que la población de este estudio comprende la edad escolar no se valorará la regulación integrada porque implica integrar el deporte como estilo de vida y es característico de la etapa adulta) que evolucionan de mayor a menor nivel de autodeterminación y hace referencia al compromiso en una actividad por las consecuencias más que por la actividad en sí misma y, por último, la desmotivación, que es el menor nivel de autodeterminación y refleja la falta de intención para comprometerse con un comportamiento.

En esta línea, se han desarrollado varios trabajos que tratan de mostrar la existencia de un vínculo negativo entre la motivación autodeterminada y el conflicto de roles sociales (Ratelle, Vallerand, Senécal \& Provencher, 2005; Senécal, Julien \& Guay, 2003; Senécal, Vallerand \& Guay, 2001). Los resultados reflejaron que, en efecto, la motivación no autodeterminada se relacionaba con un mayor conflicto de roles entre el trabajo y la familia (Senécal et al., 2001), entre la escuela y las relaciones interpersonales (Senécal et al., 2003) y entre la escuela y el ocio (Ratelle et al. 2005).

Así pues, el objetivo principal de este estudio es examinar la relación entre la percepción de conflicto o utilidad del deporte respecto a otros papeles sociales y el nivel de autodeterminación en jóvenes deportistas. Asimismo, se pretenden observar las diferencias que existen entre estas variables en función del género del deportista, pues hay que tener presente 
que la tasa de abandono no se produce del mismo modo en ambos géneros, sino que existen diferencias dependiendo de aspectos tales como el interés por el tipo de deporte, expectativas y motivaciones diferentes para la práctica deportiva, facilidad o dificultad de acceso, disposición y distribución del tiempo libre (Sicilia, 2003; Vázquez, 2001; Vázquez, Fernández \& Ferro, 2000). Tal consideración, hace necesario conocer las diferencias de opinión del género masculino y femenino entorno a estas percepciones para poder incidir sobre ellas y reducir progresivamente esta tasa de abandono.

\section{Método}

\subsection{Participantes}

La muestra de esta investigación estuvo compuesta por 1897 jugadores de baloncesto $(n=284)$, balonmano $(n=127)$, fútbol $(n=$ 1356) y voleibol $(n=130)$. Los participantes eran de género masculino $(n=1378)$ y femenino $(n=519)$, con edades comprendidas entre los 11 y los 16 años $(M=12.37 ; D T=1.48)$. Los deportistas pertenecían a equipos federados de categorías alevín $(n=934)$, infantil $(n=599)$ y cadete $(n=364)$ de la comunidad de Extremadura.

\subsection{Instrumentos}

Nivel de autodeterminación. Para valorar el nivel de autodeterminación se utilizó la versión traducida al castellano por Núñez, Martín-Albo, Navarro y González (2006) de la Sport Motivation Scale de Pelletier et al. (1995). Esta escala consta de 28 ítems precedidos de la frase «Participo y me esfuerzo en mi deporte...» que se dividen en 5 factores motivacionales. El primer factor es la motivación intrínseca (12 ítems, ej.: «Por la diversión que experimento cuando practico esta actividad»), el segundo factor es la motivación extrínseca, que incluye tres tipos de regulación: la regulación identificada ( 4 ítems, ej.: «Porque para mí es una de las mejores formas de conocer gente»), la regulación introyectada (4 ítems, ej.: «Porque me sentiría mal si no dedicase el tiempo necesario para practicarlo») y la regulación externa (4 ítems, ej.: «Porque me permite estar bien visto por la gente que conozco») y, el tercer factor, es la desmotivación (4 ítems, ej.: «No lo sé claramente, creo que mi sitio no está en el deporte»).

En relación a la consistencia interna, se utilizó el coeficiente alfa de Cronbach, mostrando unos valores de .86 para la motivación intrínseca, de .60para la regulación identificada, de .64 para laregulación introyectada, de .74 para la regulación externa y de .70 para la desmotivación. En el caso de los factores que han presentado una fiabilidad inferior a .70, como es indicado en varios estudios (Hair, Anderson, Tatham \& Black, 1998; Nunnally \& Bernstein, 1995), la validez interna puede ser aceptada debido al pequeño número de ítems que componen el factor.

Conflicto entre roles sociales. Para conocer la opinión sobre el conflicto o la compatibilidad entre el deporte y otros papeles sociales, se empleó un cuestionario adaptado de Boiché y Sarrazin (2007) el cual se ha denominado CCD (Cuestionario de Conflicto en el Deporte). El cuestionario consta de 9 ítems agrupados en 6 factores. El primer factor hace referencia a la percepción de conflicto del deporte para el colegio (1 ítem, ej.: «Si no hiciese deporte, sería mejor alumno pues tendría más tiempo para hacer los deberes»), el segundo factor refleja la percepción de conflicto del colegio para el deporte ( 1 ítem, ej: «Si no tuviese tanto trabajo escolar, tendría tiempo para hacer más deporte»), el tercer factor muestra la percepción de conflicto del deporte para pasar tiempo con los amigos (1 ítem, ej.: «Si no hiciese deporte, tendría más amigos pues tendría más tiempo para hacer cosas con ellos»), el cuarto factor refleja la percepción de conflicto de tener muchos amigos respecto a la práctica deportiva (ej.: «Si tuviese menos amigos, podría hacer más deporte pues tendría más tiempo libre»), el quinto factor hace referencia a la influencia positiva del deporte para el colegio ( 3 ítems, ej.: «Hacer deporte es una buena cosa para el trabajo escolar puesto que se aprende a organizar el empleo del tiempo») y, el sexto factor, muestra la influencia positiva del deporte para la amistad ( 2 items, ej.: «Hacer deporte es una buena cosa para la amistad, puesto que conoces a muchas personas agradables»).
La consistencia interna fue adecuada en todos los factores, obteniendo unos valores alfa de Cronbach de .81 en la percepción de conflicto del deporte para el colegio, de .78 en la percepción de conflicto del colegio para el deporte, de .84 en la percepción de conflicto del deporte para la amistad, de .86 en la percepción de conflicto de la amistad sobre la práctica deportiva, de 76 en la influencia positiva del deporte para el colegio y de .82 en la influencia positiva del deporte para la amistad. Ambos instrumentos fueron valorados con una escala Likert de 1 a 5 , donde 1 se correspondía con «totalmente en desacuerdo» y 5 con «totalmente de acuerdo» con la formulación de la pregunta.

\subsection{Procedimiento}

Antes de llevar a cabo la recogida de datos, se informó de los objetivos de la investigación y de la utilización de los resultados a los entrenadores, padres y deportistas. Posteriormente, una vez obtenidos los permisos pertinentes, se procedió a la toma de datos informando a los deportistas de que su participación era voluntaria y las respuestas serían tratadas confidencialmente. Los participantes fueron citados en los vestuarios donde solían cambiarse antes de la práctica deportiva para rellenar los cuestionarios, sin la presencia del entrenador, de manera individual y en un clima que les permitía concentrarse sin tener ningún tipo de distracción. El investigador principal estuvo siempre presente en el momento en que los sujetos completaban los cuestionarios, e insistió en la posibilidad de preguntar cualquier tipo de duda que apareciese durante el proceso

\section{Resultados}

En la Tabla 1, se presentan los valores descriptivos de las diferentes variables contempladas en este estudio. En loque se refiere a la motivación, se puede observar que la motivación intrínseca es la que muestra la mayor puntuación (4.08) contrastando en gran medida con la desmotivación, que refleja el valor más reducido (1.64).

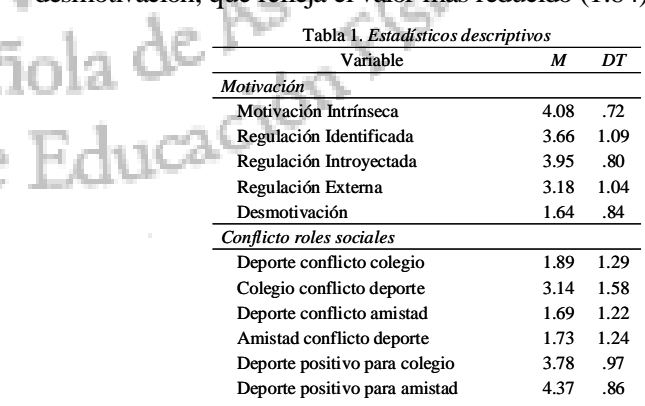

Por otro lado, en relación al conflicto o utilidad entre el deporte y otros roles sociales, se puede apreciar que las medias más elevadas corresponden a la utilidad o aspectos positivos que ejerce el deporte sobre la amistad (4.37) y sobre el colegio (3.78). En contraste, las puntuaciones más bajas pertenecen a las percepciones de conflicto entre el deporte y otros papeles sociales, excepto en el caso de la percepción de conflicto del colegio sobre el deporte, donde el valor de la media se ve incrementado (3.14).

Con el objetivo de comprobar las relaciones existentes entre los diferentes factores de la investigación, se decidió llevar a cabo un análisis de correlaciones bivariadas. Así, en la Tabla 2 aparecen reflejadas las correlaciones entre el nivel de autodeterminación y la percepción de conflicto o utilidad entre el deporte y otros roles sociales. En esta tabla, se puede contemplar que la percepción de los participantes sobre la utilidad que tiene el deporte para el colegio y la amistad se relaciona significativamente de forma positiva con los niveles más elevados de autodeterminación, motivación intrínseca y regulación identificada. Además, en el caso de la utilidad del deporte sobre el colegio, esta variable también aparece relacionada con la regulación introyectada.

En cuanto a las variables que muestran conflicto, en general se aprecia que todas estas variables se relacionan de forma positiva con las formas menos autodeterminadas de motivación, desmotivación y regulación externa, pero estas relaciones son más significativas en el caso 
Tabla 2 Correlaciones entre las variables de estudio

\begin{tabular}{|c|c|c|c|c|c|}
\hline Variable & Intrínseca & Identificada & Introyectada & Externa & Desmotivación \\
\hline Departe confl icto col egio & -.00 & $.06 * *$ & .04 & .21 ** & $.28 * *$ \\
\hline Col egio conflicto deporte & .03 & $.05^{*}$ & $.06 * *$ & $.19^{* * *}$ & $.13 * *$ \\
\hline Dep orte conflictoamistad & .00 & $.12 * *$ & $.06 *$ & $.24 * *$ & .40 ** \\
\hline Amistad conflicto deporte & .00 & $.10^{* *}$ & $.06 * *$ & $.22 * *$ & $.42 * *$ \\
\hline Deporte posit ivo para colegio & $26^{* *}$ & $.27 * *$ & $.30^{* * *}$ & $.17^{* *}$ & -.00 \\
\hline Deporte posit ivo para amistad & $29^{* * *}$ & $.26 * *$ & $.19 * *$ & $.06 *$ & $-.14 * *$ \\
\hline
\end{tabular}

Tabla 3. Análisis de varianza en función del género.

\begin{tabular}{lccccc}
\hline \multicolumn{1}{c}{ Variable } & Masculino & Femenino & $M C$ & $F$ & $p$ \\
\hline Motivación & & & & & \\
Intrínseca & $4.13 \pm .70$ & $3.96 \pm .77$ & 11.08 & 21.34 & .00 \\
Identificada & $3.74 \pm 1.07$ & $3.44 \pm 1.12$ & 34.42 & 29.10 & .00 \\
Introyectada & $4.00 \pm .79$ & $3.81 \pm .82$ & 14.72 & 22.89 & .00 \\
Externa & $3.34 \pm 1.01$ & $2.74 \pm .98$ & 135.90 & 135.26 & .00 \\
Desmoti vación & $1.71 \pm .90$ & $1.45 \pm 61$ & 26.13 & 37.44 & .00 \\
Conflicto roles sociales & & & & & \\
Deporte conflicto colegio & $1.97 \pm 1.34$ & $1.69 \pm 1.11$ & 30.48 & 18.45 & .00 \\
Colegio conflicto deporte & $3.18 \pm 1.60$ & $3.05 \pm 1.53$ & 5.45 & 2.17 & .14 \\
Deporte conflicto amistad & $1.80 \pm 1.30$ & $1.40 \pm .92$ & 61.19 & 41.82 & .00 \\
Amistad conflicto deporte & $1.84 \pm 1.32$ & $1.42 \pm .94$ & 67.60 & 44.45 & .00 \\
Deporte positivo para colegio & $3.78 \pm .98$ & $3.73 \pm .94$ & .82 & .87 & .35 \\
Deporte positivo para amistad & $4.34 \pm .87$ & $4.43 \pm .84$ & 2.95 & 3.96 & .05
\end{tabular}

o para amista

relaciones sociales (Hellín, 2003; Hicks, Wiggins, Crist \& Moode, 2001; Moreno, Martínez \& Alonso, 2006).

Como principal conclusión se puede extraer la necesidad de fomentar motivos de práctica más intrínsecos tanto en el género masculino como en el femenino, porque se ha demostrado que este tipo de motivación se asocia con una menor percepción de conflicto entre la práctica deportiva, el contexto escolar y la amistad, de forma que estos roles pueden llegar a ser compatibles en la vida de una persona, con la importancia que ello conllevaría para mantener un estilo de vida saludable durante la etapa adulta. En este sentido, ya se están desarrollando varias estrategias propuestas por autores como García-Calvo (2006), Moreno y Cervelló (2010), Moreno, Cervelló y Borges (2010) que se consideran efectivas para incrementar este tipo de motivación, las cuales deberían ser utilizadas como ejemplo para diseñar futuros programas de intervención en el entramado del deporte escolar.

\section{Referencias}

Alvariñas, M., Fernández, M. A., \& López, C. (2009). Actividad física y percepciones sobre deporte y género. Revista de Investigación en Educación, 6, 113-122.

de la desmotivación, con valores próximos o superiores al índice de correlación .30.

En la Tabla 3, se representa el análisis de varianza en función del género del deportista, donde se puede observar cómo las principales diferencias entre ambos géneros aparecen fundamentalmente en la motivación, destacando en todos los casos el género masculino frente al femenino. Asimismo, se establecen diferencias significativas en las variables que reflejan la percepción de conflicto del deporte sobre el colegio, del deporte sobre la amistad y de la amistad sobre el deporte, donde el género masculino obtiene mayores puntuaciones que el femenino.

\section{Discusión}

El objetivo principal de este estudio era examinar la relación entre la percepción de conflicto o utilidad del deporte respecto a otros papeles sociales y el nivel de autodeterminación en jóvenes deportistas, así como, conocer las diferencias que existen entre estas variables en función del género. Tras el análisis de los resultados se puede observar que la percepción de utilidad del deporte para el colegio y la amistad se relaciona positiva y significativamente con la motivación autodeterminada mientras que las percepciones de conflicto o incompatibilidad entre el deporte, el colegio y la amistad se relacionan con la desmotivación. Estos resultados ya han sido encontrados anteriormente por otros autores (Boiché \& Sarrazin, 2007; Ratelle et al., 2005; Senécal et al., 2001; Senécal et al., 2003).

En relación a las diferencias halladas en función del género, los resultados señalan la existencia de diferencias significativas en todos los tipos de motivación, destacando las puntuaciones del género masculino frente al femenino. Resultados similares han sido encontrados recientemente por Moreno, Cano, González-Cutre, Cervelló y Ruiz (2009) con una muestra de 283 deportistas federados, 141 hombres y 142 mujeres, donde hallaron mayor motivación intrínseca, extrínseca y desmotivación a favor del género masculino, lo que podría deberse a una manifestación de diferencias culturales entre ambos géneros, pero son necesarios más estudios para esclarecer tales insinuaciones.

Del mismo modo, los hallazgos de esta investigación apuntan que el género masculino percibe mayor incompatibilidad entre el deporte, el colegio y la amistad que el género femenino, mostrando diferencias significativas en tres de las cuatro variables que se abordaban en este trabajo. Este hecho puede verse influenciado porque, tal como se puede observar en la muestra, la realidad es que existe mayor número de chicos con ficha federativa que de chicas, lo que implica queel género masculino practica deporte competitivo en mayor medida que el femenino, con el compromiso y dedicación que ello conlleva, lo que le puede llevar a percibir más conflicto entre la práctica deportiva y otras actividades que la persona pueda verse obligada a desempeñar fruto de la etapa de desarrollo en la que se encuentra.

En esta línea, algunos estudios han demostrado que los chicos tienen mayores actitudes hacia actividades estimulantes, de riesgo y competitivas, mientras que las chicas muestran actitudes más favorables hacia actividades físicas que enfatizan la estética, mejoran la salud y las

Amado, D., Leo, F. M- Sánchez-Miguel, P. A Sánchez-Oliva, D., \& García-Calvo, T. (2009). Análisis del conflicto entre la práctica deportiva, el contexto escolar y la amistad en jóvenes deportistas: Importancia de padres y madres. Acción psicológica, $6(2), 45-54$

Boiché, J., \& Sarrazin, P. (2007). Motivation autodéterminée, perceptions de conflit et d'instrumentalité et assiduité envers la pratique d'une activité physique: une étude prospective sur six mois. Psychologie française, 52, 417-430.

Castillo, I. (2000). Un estudio de las relaciones entre las perspectivas de meta y otras variables motivacionales con el estilo de vida saludable en la adolescencia temprana. Tesis doctoral. Universidad de Valencia.

Deci, E. L., \& Ryan, R. M. (2000). The «what» and the «why» of goal pursuits: Human needs and the self-determination of behavior. Psychological Inquiry, 11, 227-268.

Edo, J. A. (2001). Adolescencia y Deporte en el Principado de Andorra. Tesis doctoral. Universidad de Zaragoza.

García-Calvo, T. (2006). Motivación y comportamientos adaptativos en jóvenes futbolistas. Tesis doctoral. Universidad de Extremadura.

García-Calvo, T., Leo, F. M., Martín, E., \& Sánchez, P. A. (2008). El compromiso deportivo y su relación con factores disposicionales y situacionales de la motivación. Revista Internacional de Ciencias del Deporte, 12(4), 45-58.

Guillet, E., Sarrazin, P., Carpenter, P., Trouilloud, D., \& Cury, F. (2002). Predicting persistence or withdrawal in female handballers with Social Exchange theory. International Journal of Psychology, 37(2), 92-104.

Gutiérrez, M. (2004). El valor del deporte en la educación integral del ser humano. Revista de Educación, 335, 105-126.

Hellín, P. (2003). Hábitos físico-deportivos en la Región de Murcia: implicaciones para la elaboración del currículum en el ciclo formativo de Actividades FísicoDeportivas. Tesis Doctoral. Universidad de Murcia.

Hicks, M. K., Wiggins, M. S., Crist, R. W., \& Moode, F. M. (2001). Sex differences in grade three students' attitudes toward physical activity. Perceptual and Motor Skills, 93, 97-102.

Moreno, J. A., Cano, F., González-Cutre, D., Cervelló, E., \& Ruiz, L. M. (2009). Flow disposicional en salvamento deportivo. Una aproximación desde la teoría de autodeterminación. Revista de Psicología del Deporte, 18(1), 23-35.

Moreno, J. A., \& Cervelló, E. (2010). Motivación en la actividad física y el deporte. Sevilla: Wanceulen.

Moreno, J. A., Cervelló, E., \& Borges, F. (2010). 20 estrategias para aumentar la motivación en programas de ejercicio físico. Sevilla: Wanceulen.

Moreno, J. A., Martínez, C., \& Alonso, N. (2006). Actitudes hacia la práctica físicodeportiva según el sexo del practicante. Revista Internacional de Ciencias del Deporte, 3(2), 20-43

Núñez, J. L., Martín-Albo, J., Navarro, J. G., \& González, V. M. (2006). Preliminary validation of a Spanish version of the Sport Motivation Scale. Perceptual and Motor Skills, 102, 919-930.

Pavón, A. (2004). Motivaciones e intereses de los universitarios murcianos hacia la práctica físico-deportiva. Tesis Doctoral. Universidad de Murcia

Pelletier, L. G, Fortier, M. S., Vallerand, R. J., Tuson, K. M., Briére, N. M., \& Blais, M. R. (1995). Toward a new measure of intrinsic motivation, extrinsic motivation, and amotivation in sports: The Sport Motivation Scale (SMS). Journal of Sport and Exercise Psychology, 17, 335-353.

Ratelle, C. F., Vallerand, R. J., Senécal, C., \& Provencher, P. (2005). The relationship between school-leisure conflict and educational and mental health indexes: A motivational analysis. Journal of Applied Social Psychology, 35(9), 1800-1823.

Rodríguez-Allen, A. (2000). Adolescencia y deporte. Oviedo: Ediciones Nobel.

Ryan, R. M., \& Deci, E. L. (2000). Self-determination theory and the facilitation of intrinsic motivation, social development, and wellbeing American Psychologist, 55, 68-78

Senécal, C., Julien, E., \& Guay, F. (2003). Role conflict and academic procrastination: a self-determination perspective. European Journal of Social Psychology, 33(1), 135-145.

Senécal, C., Vallerand, R. J. \& Guay, F. (2001). Antecedents and Outcomes of Work-Family Conflicts: Toward a Motivational Model. Personality and Social Psychology Bulletin, 27(2), 176-186.

Sicilia, A. (2002). La investigación sobre el pensamiento del alumnado. Una revisión desde la Educación Física. Revista de Educación, 331, 577-613.

Vázquez, B. (2001). La cultura física y las diferencias de género en el umbral del siglo XXI En J. Devís (Ed.), La educación física, el deporte y la salud en el siglo XXI (pp. 213226). Alcoy: Marfil.

Vázquez, B., Fernández, E., \& Ferro, S. (2000). Educación Física y Género: Modelo para la observación y el análisis del comportamiento del alumnado y del profesorado. Madrid: Gymnos. 